\title{
Microbial dark carbon fixation fueled by nitrate enrichment
} Joseph H. Vineis, Ashley N. Bulseco, and Jennifer L. Bowen

Department of Marine and Environmental Sciences, Marine Science Center, Northeastern University, Nahant, Massachusetts.

Corresponding Author:

Joseph $\mathrm{H}$. Vineis

MSC(Marine Science Center)

Nahant, MA 01908

508-274-8236

jvineis@gmail.com

Abstract

Anthropogenic nitrate amendment to coastal marine sediments can increase rates of heterotrophic mineralization and autotrophic dark carbon fixation (DCF). DCF may be favored in sediments where organic matter is biologically unavailable, leading to a microbial community supported by chemoautotrophy. Niche partitioning among DCF communities and adaptations for nitrate metabolism in coastal marine sediments remain poorly characterized, especially within salt marshes. We used genome-resolved metagenomics, phylogenetics, and comparative genomics to characterize the potential niche space, phylogenetic relationships, and adaptations important to microbial communities within nitrate enriched sediment. We found that nitrate enrichment of sediment from discrete depths between 0-25 cm supported both heterotrophs and chemoautotrophs that use sulfur oxidizing denitrification to drive the Calvin-BensonBassham (CBB) or reductive TCA (rTCA) DCF pathways. Phylogenetic reconstruction indicated that the nitrate enriched community represented a small fraction of the phylogenetic diversity contained in coastal marine environmental genomes, while pangenomics revealed close evolutionary and functional relationships with DCF microbes in other oligotrophic environments. These results indicate that DCF can support coastal marine microbial communities and should be carefully considered when estimating the impact of nitrate on carbon cycling in these critical habitats.

Importance

Salt marshes store carbon at one of the fastest rates of any blue carbon system and buffer coastal marine waters from eutrophication. Dark carbon fixation (DCF) conducted by microbes within the sediment can influence the carbon storage capacity, but little is known about the ecology or genomic potential of these organisms. Our study identifies a potential niche space for several functionally distinct groups of chemoautotrophs which primarily use sulfur oxidizing denitrification to fuel DCF under high nitrate concentrations. These findings fill an important gap in our understanding of microbial contributions to carbon storage within salt marsh sediments and how this critical blue carbon system responds to anthropogenic nitrate enrichment.

Introduction

Salt marsh sediments store carbon at a higher rate than any other coastal marine

47 habitat (1) and the accumulation of carbon is influenced by diverse heterotrophic and 
autotrophic microbial engineers inhabiting the sediment. Macrophyte communities dominate the surface of the salt marsh platform and contribute significant amounts of carbon in the form of roots, shoots, and exudates $(2,3)$. Sulfate reduction is the primary respiratory process used by the heterotrophic microbial community to decompose macrophyte derived carbon sources (4), resulting in a loss of photosynthetically produced carbon and the production of large pools of reduced sulfur and ammonium. These pools of reduced compounds in turn can be used by chemoautotrophic microbes to fix inorganic carbon in the absence of light using dark carbon fixation (DCF) (5-7). Near-shore and shelf sediments are among the most important contributors to oceanic carbon fixation (0.29 $\left.\mathrm{Pg} \mathrm{C} \mathrm{yr}^{-1}\right)$ (8) and primary production (9). Thus, chemoautotrophic microorganisms could represent a significant contribution to carbon storage in salt marsh sediments, based on evidence from molecular genomics (10), carbon isotopes $(10,11)$, and relevance in other marine systems $(8,12)$.

Many chemoautotrophs inhabiting marine sediments primarily use either the Calvin Benson Bassham (CBB) or the reductive TCA (rTCA) cycle to fix inorganic carbon (7) using energy derived from the oxidation of reduced sulfur or ammonium (7). Facultative sulfide oxidizing bacteria (SOB) can generate energy for DCF using the multienzyme sox pathway or through a reverse-acting dissimilatory sulfite reductase (rDSR) coupled with an adenosine 5'-phosphosulfate (APS) reductase and an ATPsulfurylase that completely oxidizes reduced sulfur to sulfate $(5,13-16)$. Nitrate can be used as the terminal electron acceptor for the oxidation of reduced sulfur in SOB within marine sediments and increased nitrate availability can simultaneously stimulate production of sulfate and nitrous oxide (17). Current knowledge of the physiology, phylogeny, and niche space of microbial populations that govern DCF is limited, hampering our ability to accurately predict how anthropogenically driven changes in climate and biogeochemistry will influence carbon storage in coastal marine sediments. Examining the genomic capacity of microbes in salt marsh sediments exposed to high concentrations of nitrate will provide important insight on the relative importance of autotrophic vs. heterotrophic nitrate reduction in coastal systems exposed to excess nutrients.

To improve our understanding of microbial contributions to carbon cycling within coastal sediments, we reconstructed draft genomes from metagenomic data (MAGs) derived from a previous $\mathrm{NO}_{3}{ }^{-}$enrichment experiment (18). The recovery of draft genomes allows us to resolve several outstanding unknowns regarding DCF within salt marsh sediments including: 1) Common metabolic strategies and traits potentially important to defining the niche space; 2) The vertical niche space boundaries among chemoautotrophs; and 3) phylogenetic and functional relationships with other chemoautotrophs. To address the first two unknowns, we characterized MAGs across a sediment depth profile and identified genes related to DCF, decomposition of organic carbon, and sulfur and nitrogen cycling. To address the third unknown, we used a pangenomic approach to identify genes that are potentially important to inhabiting coastal sediments.

Methods:

92 
102

103

104

105

106

107

108

109

110

111

112

113

114

115

116

117

118

119

120

121

122

123

124

125

126

127

128

129

130

131

132

133

134

135

136

137

138

139

We collected 27 sediment samples from a flow through reactor (FTR) experiment described previously $(18,19)$, where every effort was made to prevent the influence of light and oxygen. We collected three replicate sediment cores from the tall ecotype of Spartina alterniflora.Sediment from "shallow" (0-5 cm), "mid" (10-15 cm), and "deep" (20-25 cm) regions from each core were sectioned and homogenized independently under anoxic conditions and placed into two separate FTRs $(n=18)$. Nine FTRs received $500 \mu \mathrm{M} \mathrm{K}^{15} \mathrm{NO}_{3}{ }^{-}$in sterile sea water ("N-enriched") and the other nine received sterile sea-water alone ("unenriched") at a flow rate of $0.08 \mathrm{~mL} \mathrm{~min}^{-1}$. After 92 days, we collected sediment from each of the reactors. Sediment was also collected from the homogenized depth sections of each core prior to the beginning of the FTR experiment ("pre-enriched"). An additional 6 samples were collected from the top $5 \mathrm{~cm}$ of sediment from two creeks located in the same marsh, part of the Plum Island Long Term Ecological Research (PIE-LTER) marsh complex ("in-situ"). The in-situ samples were collected in May, July, and September from a N-enriched and a paired reference creek area dominated by Spartina alterniflora and described in Kearns et al. (2016). Additional metadata can be found in Table S1.

\section{MAG reconstruction and quality evaluation}

Details of metagenomic DNA extraction, sequencing library preparation, quality filtering, and metagenome assembly are included in supplemental methods. We reconstructed draft genomes for each assembly and summarized the quality of each metagenomic assembled genome (MAG) using Anvi'o v5 (20). We do not report the assembly statistics or MAGs from the in-situ samples due to a highly fragmented assembly.

Identification of carbon fixation pathways and nitrogen, sulfur, and organic carbon cycling potential among MAGs

Functional annotation of genes related to carbon, nitrogen, and sulfur cycling was carried out using DRAM (21). Confirmation of DRAM annotation and genome queries of additional functional genes were conducted using HMM models obtained from Fungene (22) and the Carbohydrate Active Enzymes database (CAZy) (23). The search for additional functional genes using Fungene and CAZy HMM models was conducted using HMMER and facilitated by Anvi'o. Metabolic potential was also estimated for each of the genomes according to the RAST genome annotation tool (24). We estimated the percentage of genes in two well-known carbon fixation pathways, the Calvin-BensenBassham (CBB) and reductive TCA (rTCA) cycle, according to the detection of several genes in each of the pathways during DRAM annotation and confirmed using RAST. We used phylogenetic confirmation to ensure the accuracy of assigning CBB and rTCA to each MAG (Supplemental). The HMM models we used and a table containing gene names and links to HMM models are reported in Table S2 and custom Anvi'o HMMs are available here (https://github.com/jvineis/Reactor-metagen/).

\section{Phylogenetics of estuary environmental genomes}

We built a phylogenetic tree of FTR MAGs and all MAGs in the Genomes from Earth's Microbiomes (GEM) (25) database that were recovered from marine intertidal and salt marsh habitats to identify evolutionary relationships among the FTR MAGs and 
other coastal environments. Details of the MAGs, samples included, and the methods employed can be found here https://github.com/jvineis/Reactor-metagen/.

MAG taxonomic classification, dereplication and relative abundance

We used the GTDB-Tk classifier (26) to assign a taxonomic classification to each MAG (GTDB) (27). Average nucleotide identity (ANI) among all MAGs was computed using pyani (28) employed with Anvi'o v7. MAGs with an ANI of $95 \%$ over $90 \%$ of their genome were de-replicated to estimate their relative abundance (RA) in each of the samples. The contigs of the dereplicated collection of MAGs were concatenated into a single collection and each short-read dataset was mapped to this collection using bbmap (29). We used samtools (30) to filter, sort, and index the mapping results. MAG relative abundance in each sample was calculated to allow for comparison of relative abundance across and within samples according to the following equation:

$$
\text { Relative abundance }=\left(\frac{\text { minimum } \# \text { of reads in any sample }}{\# \text { of reads in target sample }}\right) \times\left(\frac{\text { bases mapped to } M A G}{\text { genome length }}\right)
$$

We estimated the occurrence of each MAG within each experimental group by dividing the sum of the relative abundance of a MAG within an experimental group by the total relative abundance observed across all experimental groups.

\section{Pangenomics of Chlorobium and Sedimenticola}

We used reference genomes for a pangenomic analysis to identify functional and evolutionary relationships among the chemoautotrophs we recovered. We selected Chlorobium and Sedimenticola as representative chemoautotrophs that employ rTCA and CBB respectively, because we assembled multiple Chlorobium and Sedimenticola MAGs. There are also several well studied reference genomes available for comparison. We obtained 20 Chlorobium and 14 Sedimenticola reference genomes from IMG and NCBI with completion estimates above $50 \%$ and redundancy below $10 \%$ according to the collection of single copy genes used by Anvi'o v7. We employed a pangenomic analysis, including the identification of gene clusters, and ANI among the MAGs and reference collections using Anvio v7.

\section{Data availability and reproducibility}

We provide open access to each of the bioinformatic steps here: https://github.com/jvineis/Reactor-metagen/. The files required for visualization of contig coverage across samples and placement into respective MAGs are located here: https://figshare.com/articles/dataset/Anvio Files/12034611. Raw sequence reads are archived on the MG-RAST server under Project Numbers mgp84173 and mpg99679.

Results:

MAG reconstruction

Genome reconstruction of the 27 metagenomic samples from the FTR experiment produced a collection of 113 MAGs that were greater than $48 \%$ complete and less than $7 \%$ redundant according to a collection of 71 single copy genes, with average completion and contamination estimates of $79.0 \%$ and $1.8 \%$ respectively. With 
184

185

186

187

188

189

190

191

192

193

194

195

196

197

198

199

200

201

202

203

204

205

206

207

208

209

210

211

212

213

214

215

216

217

218

219

220

221

222

223

224

225

226

227

228

229

the exception of assembled rRNA genes (Table S3), 44 of the MAGs met all the criteria of high-quality MAGs (31). Mean coverage estimates for the high-quality MAGs, based on mapping reads used to assemble the MAGs, ranged from $5.7-53.9$ with an average of 15.5 (Table S1). The number of MAGs recovered from each of the samples ranged from 0-15 (Table S1) and the vast majority (93 of 113) were recovered from the Nenriched samples. Reconstruction of MAGs was most successful from mid depth and deep samples, as opposed to shallow samples, with a total of 33 and 42 MAGs, respectively. The percentage of the metagenome mapping back to our collection of MAGs was also highest in N-enriched samples, where read recruitment ranged from 6.5 $-27.3 \%$, with a mean of $13.0 \%$. We did not observe more than $2.4 \%$ read recruitment in either the pre, unenriched, or in-situ samples (Table S1).

\section{Chemoautotrophic functional groups, sulfur oxidation, and denitrification}

We assessed chemoautotrophy using CBB and rTCA within each MAG using aclA/aclB and cbbS/cbbL respectively and phylogenetic confirmation of the genes for each MAG are shown in Fig. S1. To provide additional evidence for chemoautotrophy in each MAG, we calculated the proportion of the 10 and 9 genes assessed for pathway completion in the rTCA and CBB pathways respectively (Fig. 1, Fig. S1, Table S2). We did not place a threshold on the number of genes required to be considered a competent chemoautotroph and instead relied on the presence of $a c / A / a c / B$ and $c b b S / c b b L(32,33)$. According to the presence of these genes, we found evidence of inorganic carbon fixation potential coupled with denitrification and sulfur oxidation in 59 of 113 MAGs (Fig. 1). Genomic potential indicative of denitrifying sulfur oxidation was detected within five distinct functional groups of chemoautotrophic MAGs (Fig. 1.).

MAGs of three of the functional groups (Thiohalomonadales/SZUA (TS), Sedimenticola (S), and Magnetovibrio/Rhodspirallales (MR)) contained the potential for CBB, according to the presence of $c b b L$ or $c b b S$ (Fig. 1). These groups could be broadly classified as denitrifying sulfur oxidizers, containing genetic evidence for at least one marker gene in the denitrification pathway: nitrate reductase (narGHI/napAB), nitrite reductase (nirSK), nitric oxide reductase (norCB), and/or nitrous oxide reductase (nosZ) (Table S4). We identified complete denitrification potential in 17 MAGs identified with CBB potential in these three functional groups (Table S4). Sulfur oxidation potential of the MAGs within these three CBB functional groups was commonly characterized by the presence of the SOX gene complex (SOXABXYZ), dissimilatory sulfite reduction (dsrAB), APS reductase (aprAB), and ATP sulfurylase (sat). We graphically characterized the cooccurrence of these genes for a Sedimenticola MAG in the S group to demonstrate the interconnectivity of the sulfur, nitrogen and carbon related metabolic potential (Fig. 2).

The Sulfurimonas/Sulfurimonadaceae group (SUL) members contained genomic potential for carbon fixation via the rTCA cycle, sulfur oxidation via a soxCDYZ complex, and sulfate assimilation through sulfate adenylyltransferase (cysDN) (Fig. 1). The SUL group also contained genes for either dissimilatory nitrate reduction to ammonia (nrfAB) or nitrogen fixation $(\mathrm{nifH})$ in addition to at least one gene in the denitrification suite (Table S4). We detected evidence of the rTCA cycle among the Chlorobiaceae group $(C L)$, which also contained genes for dissimilatory sulfate reduction (dsrAB) and (aprAB) and nitrogen fixation ( $\mathrm{nifH}$ ). Based on the genomic characterization of the CL group, they are likely photoautotrophs with the potential capacity for mixotrophic growth. We 
graphically characterized gene pathways for a representative Chlorobium MAG demonstrating this mixotrophic capacity (Fig. 2).

MAGs representing each of the four chemoautotrophic functional groups

\section{Phylogeny, taxonomic resolution, and average nucleotide identity among chemoautotrophic functional groups}

The phylogenetic reconstruction of estuary and intertidal MAGs containing a sufficient number of ribosomal protein encoding genes included 2706 MAGs from the Genomes from Earth's Microbiomes (GEM) database and 96 recovered in this study. Overall, the tree contained 54 different phyla from 2688 Bacterial and 114 Archaeal genomes. The most species rich phyla included Proteobacteria, Bacteroidota, and Actinobacteriota, which contained 1099, 590, and 274 MAGs respectively (Fig. 3). The MAGs recovered from $\mathrm{N}$-enriched samples were distributed across six of the 54 phyla and included Bacteroidota, Campylobacterota, Deslufuromonadota, Nanoarchaeota, Patescibacteria, and Proteobacteria (Table S3), with 71 of the FTR MAGs assigned to Proteobacteria. The unenriched MAGs were distributed across the same phyla, except for Desulfuromonadota (Table S3).

The carbon fixation groups we identified were distributed across three phyla (Fig. 3). The four non-redundant Chlorobium MAGs from the unenriched samples were all located on adjacent leaves within the Bacteroidota and represented the only genomes from this genus in the collection of marine intertidal and estuary samples in our analysis (Fig. 3). They were most closely related to Chlorobium recovered from a chemocline in the Black Sea (see pangenomic results). Four of the Sulfurimonas MAGs with the potential to fix carbon using the rTCA cycle were found on a single branch among 22 Campylobacterota along with a reference Sulfurimonas MAG recovered from the metagenome of inlet seawater (JGI project ID: 3300024257). Three of the Sulfurimonas MAGs recovered from mid and deep sediments were redundant. The nine nonredundant Sedimenticola were in the same region of the tree as the Thiohalomonadales/SZUA group and there were other MAGs from marine systems located on adjacent leaves. There was a clear phylogenetic split of the MAGs classified as Thiohalomonadales and SZUA. The SZUA were unique MAGs whose closest relatives were isolated from a study of hydrothermal vent communities of the Guaymas Basin (34). The Magnetospiraceae and Rhodobacteraceae that formed a cluster based on functional annotation (Fig. 1) were phylogenetically distinct (Fig. 3). The 
Rhodobacteraceae recovered from the mid and deep depths of $\mathrm{N}$-enriched sediment from core 1 (N1M-Bin_22, N1D-Bin_9) were collapsed into one MAG based on our ANI thresholds (Table S5, Table S6).

The phylogeny of the MAGs that were not classified as chemoautotrophs included eight different phyla (Fig. 3). The Ignavibacteriaceae were recovered from preenriched, unenriched, and N-enriched conditions; the Thermoanaerobaculia, Desulfobacterota, and Bathyarchaeia were recovered from unenriched reactors; and several others including Nanoarchaeia, koll-22, and Xanthomonadales were recovered exclusively from $\mathrm{N}$-enriched reactors. The eight MAGs classified as koll-22 were all found to represent a single redundant collection of MAGs and were classified as $\mathrm{BRH}$ c54, a genus level classification assigned to a MAG recovered from the porewater of opalinus clay rock (35). The collection of 10 Ignavibacteriaceae MAGs formed a tight phylogenetic cluster that included reference MAGs from marine wetlands. All Ignavibacteriaceae MAGs were found to be unique non-redundant populations and two of the MAGs (S2M-Bin_6, S2M-Bin_2) were isolated from the same unenriched sample (unenriched core 2). Seven of the Ignavibacteriaceae MAGs were classified as BMS3ABIN03, a genus isolated from sub-seafloor massive sulfide deposits (36). Two MAGs were classified as Archaeae, and one as a member of the candidate phyla Patescibacteria (Fig. 3).

We recovered 97 unique MAGs from the original collection of 113 MAGs according to the threshold of $95 \%$ identity over $90 \%$ of the length of the MAG (Table S3, Table S5, Table S6).

\section{MAG relative abundance among $\mathrm{N}$-enriched reactors}

Most of the 97 dereplicated MAGs were detected in multiple depths within independent cores, but we observed trends in the abundance that were apparently dependent on depth and $\mathrm{N}$ enrichment (Fig. 4). For example, N2S-Bin_4 was detected at each depth in all $\mathrm{N}$-enriched cores, but was not detected in the pre-enriched, unenriched cores, or in-situ samples. This MAG also contained seven other MAGs in the dereplicated cluster (Table S3).

The MAGs recovered from the $\mathrm{N}$-enriched samples were rarely detected in the pre-enriched or unenriched reactors and in-situ samples (Fig. 4). Exceptions to this observation included N3M-Bin_11 and N3D-Bin_5, which are both members of the (TS) group. We also observed that the reads recruited by the N-enriched MAGs were depth dependent. For example, most of the MAGs recovered from the shallow sediments were primarily detected in the shallow samples of our collection, rather than mid or deep samples (Fig. 4). However, MAG N1D-Bin_1 was recovered from a deep fraction but recruited more reads from almost all the other $\mathrm{N}$-enriched samples (Fig. 4). This is likely due to the failure to dereplicate this MAG from N3S-Bin_13. Although N1D-Bin_1 and N3S-Bin_13 shared 99\% ANI, the length of the genome alignments was $72 \%$ and $49 \%$ for each of the pairwise alignments (Table S5, Table S6). The functional similarity (Fig. 1) and taxonomic classification (Table S3) serve as additional lines of evidence that these two MAGs should be considered the same organism, which was recovered from the same core at shallow, mid, and deep fractions. Several additional N-enriched MAGs could also be detected at multiple depth fractions of the same core. For example, N1MBin_3 could be detected in three samples (N1S, N1M, and N1D) (Fig. 4). 
Apart from the $\mathrm{CL}$ group, which were only detected in the unenriched reactor samples, MAGs within the chemoautotrophic functional groups were detected at each depth fraction in all three cores but recovered primarily from the mid and deep depths, where 33 and 42 unique MAGs were recovered respectively (Fig. 4). Members of the chemoautotrophic functional groups were also among the most abundant MAGs in the $\mathrm{N}$-enriched samples (Fig. 4). MAGs within the S functional group were not recovered from the deep sediments and no SUL members were recovered from the shallow sediments (Fig. 4). However, we did detect MAGs representing these groups through read mapping within each of the depths, as shown in Fig. 4. This trend of co-occurrence among MAGs within each functional group was observed within each of the $\mathrm{N}$-enriched samples. The heterotrophic groups were also detected in each of the depths of $\mathrm{N}$ enriched samples. Although the k22 group appears to be absent, members of the dereplicated cluster represented by N2S-Bin_4 were recovered from mid depths (e.g. N3M-Bin_4) (Fig. 1).

\section{Pangenomics of Chloribium and Sedimenticola}

The Chlorobium pangenomic analysis included three FTR MAGs and 20 reference genomes classified as $C$. phaeobacteroides, $C$. chlorochromatii, $C$. phaeovibriodes, C. limicola, and C. ferrooxidans. We identified a total of 5803 gene clusters and 239 core single copy genes. The three FTR Chlorobium MAGs formed a cohesive cluster based on phylogenomic and gene cluster content (Fig. S2) and shared the most gene clusters with marine and hot spring derived strains. A Chlorobium phaeobacteroides reference genome recovered from a chemocline located $100 \mathrm{~m}$ below the surface of the Black sea (GCA_000020545) was most closely related to the FTR Chlorobium MAGs according to gene cluster content and phylogeny determined from single copy core genes (Fig S2). The ANI among the FTR Chlorobium MAGs and the Black sea reference was above $80 \%$. We identified 443 gene clusters that were unique to the FTR Chlorobium MAGs and C. phaeobacteroides. Genes within this group encoded proteins related to cofactor and vitamin metabolism, lipid metabolism, ATP synthesis, central carbohydrate metabolism, and a photosystem P840 reaction center cytochrome c551. We also identified a collection of 104 gene clusters that were unique to only the FTR Chlorobium MAGs (Fig S2, Table S7). Functions encoded by genes within this cluster included multiple proteins for F-type ATP synthesis and heme biosynthesis.

We chose Sedimenticola as the representative genus for MAGs that contain the genetic capacity for the rTCA cycle. The pangenomic analysis of Sedimenticola included four FTR MAGs and 14 reference genomes including S. selenatireducens, S. thiotaurini, and S. endophacoides. Pangenomic analysis identified a total of 6811 gene clusters. We identified 262 single copy gene clusters that we used to determine phylogeneomic relationships among genomes. We found that N1M-Bin_16 was most closely related to two S. selenatireducens reference genomes (GCA_002868805 and GCA_000428045) isolated from marine sediment (Fig S2). The ANI between N1MBin_ 16 and these genomes was above $88 \%$. The proportion of gene clusters shared with N1M-Bin_16 and GCA_002868805 was highest, but S. thiotaurini and other Sedimenticola were also all above 80\% (Fig. 5). N1M_Bin-2 and N2S_Bin-3 formed a cluster with S. thiotaurini (GCA_007713595) and ANI between this reference genome 
and the FTR MAGs was above $95 \%$ in both cases. We identified 220 accessory gene clusters that were found only in FTR MAGs, S. selenatireducens, S. thiotaurini, and unclassified Sedimenticola derived from marine environments. Genes within this cluster encode a wide variety of functions including those related to glyoxolate bypass, glycolysis and rTCA (Table S7). These clusters also contained genes important for cofactor and vitamin metabolism.

\section{Discussion:}

While the importance of chemoautotrophy in coastal sediments has been recognized for decades (11), few studies have employed genome resolved metagenomic approaches to study them, leaving gaps in our ability to understand biogeochemical processes of the sediment. Our experiment, with high-nitrate, dark, and anoxic conditions applied to sediments for 92 days with no organic matter input, created a niche for chemoautotrophy in each of the three depths examined here. Our observations of chemoautotrophs were stable across three independently collected cores from sediment inhabited by the tall ecotype of Spartina alternifora. The reconstruction of 113 MAGs from these sediments enabled us to address three outstanding unknowns about microbial chemoautotrophs in salt marshes, 1) their metabolic flexibility and pathways for DCF, 2) the potential niche space within the top 25 $\mathrm{cm}$ of the sediment, and 3) the functional and phylogenetic relationship to microbes inhabiting oligotrophic environments. This study provides much needed genomic insight to improve our understanding of the relevance of DCF to carbon cycling in marine coastal systems and the evolution of DCF within oligotrophic environments more broadly.

We identified three distinct functional strategies for DCF within the FTR cores including CBB driven by denitrifying sulfur oxidation, rTCA driven by denitrifying sulfur oxidation, and rTCA fueled by photoautotrophy despite extremely low light levels (Fig. 1). The use of multiple electron acceptors by the collection of chemoautotrophic MAGs in each of the functional groups could provide an important adaptation to inhabiting the top $25 \mathrm{~cm}$ of marsh sediment. Salt marsh sediments contain steep gradients in oxygen $(37,38)$ and nitrate $(19,39)$, and a sulfate to sulfide transition zone $(40)$, all of which are influenced by diurnal tidal inundation that provides a re-supply of fresh organic matter and nutrients (41). The biogeochemistry of the sediment is also influenced by the release of oxygen and carbon from plant roots $(42,43)$ and animal burrows, providing additional structure and permeability to sediment that enhances chemoautotrophic growth (44). These conditions also favor diverse heterotrophic bacteria that primarily rely on sulfate reduction due to the low availability of oxygen and nitrate $(45,46)$. However, the quality of carbon decreases with depth following the mineralization of simple carbon by heterotrophs, reducing the availability of favorable carbon sources used by the heterotrophic community. These conditions present a potential niche space for chemoautotrophic bacteria.

The chemoautotrophic MAGs recovered from the $\mathrm{N}$-enriched reactors contained genes for complete dentification and cytochromes that require oxygen as the terminal electron acceptor among functionally diverse groups of bacteria (Fig. 1, Fig. S3). The ability to use both nitrate and oxygen as terminal electron acceptors, while oxidizing 
414 multiple reduced forms of sulfur to produce their own carbon, provides potentially important adaptations to an environment with rapidly changing electron acceptor concentrations and low availability of simple forms of carbon (47). The presence of the different functional groups using the same core metabolism of denitrifying sulfur oxidation suggests that there may be a significant physical niche space allowing for the accumulation of diverse microbes contributing to DCF $(48,49)$.

The presence of these chemoautotrophic groups within the sediment inhabited by the tall ecotype of $S$. alterniflora has important consequences for the ecology of the vegetation and surrounding waters. Many of the chemoautotrophic MAGs are complete denitrifiers (Fig.1, Table S4) and most contain the gene for nitrous oxide reductase (nosZ), which provides the potential to release inert $\mathrm{N}_{2}$ from the sediment as opposed to the potent greenhouse gas, $\mathrm{N}_{2} \mathrm{O}$. The capacity to oxidize reduced sulfur from the sediment is important to the health of $S$. alterniflora due to the toxicity of high sulfide concentrations (50). The carbon storage potential of the sediment could also be affected if appreciable amounts of carbon are being fixed by the chemoautotrophs. Within anoxic sediments below $10 \mathrm{~cm}$, fresh inputs of carbon are likely limited, so the chemoautotrophic necromass may be an important carbon source for the heterotrophic community (51). Cycling of carbon between chemoautotrophic and heterotrophic groups could provide a mechanism to preserve and cycle carbon stored in the system.

The canonical denitrifying MAGs, including members of the Colwellia, Motiliproteus, and novel genera within the Kangiellaceae family, recovered from the $\mathrm{N}$ enriched sediment were largely complete denitrifiers (Fig. 1). However, we observed the carbon sources potentially used by most heterotrophic denitrifiers were the same as the chemoautotrophic denitrifying sulfur oxidizers, including chitin and polyphenolics. Three Colwellia redundant MAGs recovered from three different depths and two separate cores contained evidence for more extensive use of plant derived carbon, primarily from the decomposition of hemicellulose (Fig. S3). The lack of a significant carbohydrate usage profile among most of the heterotrophic denitrifiers (Fig. 1, Fig. S3) provides evidence that they may rely on more simple forms of carbon derived from the chemoautotrophic necromass or through syntrophic interactions. Coupling between chemoautotrophic carbon fixation fueling heterotrophic communities can be found around hydrothermal vents (52), and in benthic freshwater ecosystems (53). Recent evidence also suggests that low abundance chemoautotrophic members of the community can support heterotrophic communities within deep sea oligotrophic sediment through syntrophic interactions and metabolic handoffs (54). Quantifying the flow of organic carbon among the heterotrophs and autotrophs in the sediment will provide important insights about the flow and retention of carbon within these blue carbon systems.

While chemoautotrophs may be low abundance members of the unenriched community (Fig. 4), the findings here suggest that there may be conditions in the heterogeneous vegetated salt marsh platform where they are abundant. The relative abundance estimates, and average nucleotide identity (ANI) of MAGs obtained from the three independent depth profiles indicates that some populations of chemoautotrophs spanned more than one depth fraction of the vertical profile and/or multiple cores (Fig.4, Table S3). Support for the presence of the same population represented by an individual MAG includes 1) the observation that redundant genomes were reconstructed 
460 within two or more $\mathrm{N}$-enriched sample cores and/or depths and 2) read mapping that indicates detection of the same MAG within multiple samples (Fig. 4). Although we did not have the required coverage for a detailed strain analysis (55), the level of nucleotide similarity $(95 \%)$ and length $(90 \%)$ thresholds used to evaluate redundancy among our collection of MAGs is strong support for the occurrence of highly similar genomes. For example, two Thiohalomonadaceae MAGs, recovered from shallow and mid-depth sediments contained $99.9 \%$ ANI at the nucleotide level over $98.8 \%$ of the genome (Table S5). Sulfurimonas MAGs were also recovered from multiple cores and contained similar alignment length percentages and ANI. These findings suggest that some populations may be large and capable of inhabiting different niches within the sediment. Sulfurimonas is known to contain organisms that are metabolically plastic and ubiquitous across the globe (56). Additional sequencing of the $\mathrm{N}$-enriched reactor samples or additional enrichment experiments would be useful to identifying the strain level variation of these communities.

Gammaproteobacteria are widespread and active members of the microbial communities inhabiting marine sediments (10) and the MAGs recovered from the Nenriched reactors expands our understanding of the functional metabolism that may be important to their ubiquitous nature. The experimental nitrate amendment allowed us to recover chemoautotrophic members of this phylum that were undetectable in the unenriched FTR or in-situ samples but represented an average estimate of $13 \%$ of the total community within nitrate enriched sediment. Our evaluation of the phylogenetic breadth of environmental genomes recovered from intertidal marine sediments identified 54 different phyla and six of these phyla are represented by MAGs in the N-enriched reactors (Fig. 3). This points to a relatively narrow phylogenetic group that are enriched by these conditions and this finding is confirmed by the reduced alpha diversity estimates for these samples observed by Bulseco et al. (19). Except for Chlorobium, the presence of close phylogenetic relatives within coastal marine systems indicates that the MAGs recovered here are not necessarily limited to salt marsh habitats. The chemoautotrophic MAGs in this study were distributed among 12 different families and many of these families have evolutionary relationships to metabolically diverse microbes inhabiting oligotrophic environments. For example, the families identified as SZUA-229 and SZUA-152 are Gammaproteobacteria originally recovered from oxic subseafloor sediments adjacent to a cold water aquifer (57). The Sulfurimonas potentially conduct DCF in salt marsh sediments (58), deep sea hydrothermal vents (59), and pelagic redox clines (60). The Sedimenticola are related to inhabitants of other oligotrophic environments including hydrothermal vents (61) and marine sediment (62). Unenriched FTR sediments also contained Chlorobium, closely related to another species inhabiting a chemocline in the Black Sea $(63,64)$. While the taxonomic relationship among the chemoautotrophic MAGs in this study is consistent with ubiquitous chemoautotrophic Gammaproteobacteria in marine systems, this study improves our ability to understand their underlying functional strategies.

To identify genomic features that are important for inhabiting such diverse environments, we conducted pangenomic analysis of the Chlorobium and Sedimenticola MAGs. Gene clusters shared among the Chlorobium MAGs recovered here with the $C$. phaeobacteroides strain recovered from the Black Sea, included a P840 reaction center and an F-type ATPase, which harvest light and provide proton 
506

regulation for the cell, respectively. We also identified unique gene clusters orthologous to vitamin and cofactor metabolism, glycan and lipid metabolism, and energy production (Table S7). These functional categories can be used to define differences between oligotrophic and eutrophic environments (65) and are important to determining the flow of carbon between soil organic matter and the microbial community (66). Oligotrophic conditions and a strong redox cline are two defining characteristics of the Black Sea chemocline as well as the sediment in our FTR experiment $(19,67)$, suggesting that these are two important characteristics of the Chlorobium niche space.

Pangenomics of Sedimenticola genomes revealed a cluster of genes shared among all Sedimenticola recovered from marine environments. The cluster contains genes related to carbohydrate and amino acid metabolism, metabolism of cofactors and vitamins, and aromatics degradation (Table S7). Strains of Sedimenticola recovered from salt marshes show improved growth in the presence of amino acids (62) while the originally described strain, Sedimenticola selenatireduces, couples the oxidation of aromatic compounds to selenate respiration (68). Interestingly, the Sedimenticola recovered from the FTR experiment shared the most gene clusters in the pangenome with S. selenatireducens. These findings highlight the complexity of microbial carbon cycling that can be recovered using genome resolved metagenomics and presents many questions about the nature of the microbial taxa recovered from $\mathrm{N}$-enriched systems.

The MAGs recovered from these nitrate enriched FTRs improve our understanding of DCF within vegetated coastal marine systems and other oligotrophic environments. Our research expands the known metabolic flexibility among Gammaproteobacteria inhabiting marine and oligotrophic sediments, extends our understanding of the niche space and population sizes of chemoautotrophs, and provides the phylogenetic context of members of the microbial community enriched by nitrate. Few chemoautotrophic genomes have been recovered from metagenomic datasets and identification of a significant niche space within salt marsh sediments will renew interest in understanding their contribution to nitrogen, sulfur, and carbon cycling. The potential for using oxygen and nitrate as a terminal electron acceptor and the evidence for chitin mineralization requires further investigation, but the observation of this metabolic potential across multiple chemoautotrophic functional groups suggests that chemoautotrophy and/or mixotrophy may be an important feature of microbial life in nitrate enriched sediments. In-situ biogeochemical measurements of chemoautotrophy paired with targeted cultivation and enrichment represent two complimentary methodologies that should be used to assess DCF relevance in these critically important coastal carbon storage systems.

\section{Acknowledgements}

We acknowledge the Bay Paul Center at the Marine Biological Laboratory for sequencing support. Funding or this project was provided by an NSF CAREER Grant to JLB (DEB1350491) and an NSF DDIG to JLB and ANB (Award \# 1701748). The original FTR experiments were supported by a Woods Hole Sea Grant award (Project \# NA140AR4170074 Project R/M-65s to Anne Giblin) and a Ford Foundation pre-doctoral fellowship to ANB. Samples were collected from the Plum Island Ecosystems LTER, which is supported by NSF (OCE 0423565, 1058747, and 1637630) and from the NSF 
552

553

554

555

556

557

558

559

560

561

562

563

564

565

566

567

funded TIDE project (DEB 1902712). Samples from this project were processed with equipment purchased under an NSF FMSL grant to Northeastern University (DBI 1722553). This publication is number TBD from the Marine Science Center.

\section{References}

1. McLeod E, Chmura GL, Bouillon S, Salm R, Björk M, Duarte CM. A blueprint for blue carbon: Toward an improved understanding of the role of vegetated coastal habitats in sequestering CO2. Front Ecol Environ. 2011; 9(10): 552-560.

2. Vernberg JF. SALT-MARSH PROCESSES : A REVIEW. Environ Toxicol Chem. 1993; 12: 2167-2195.

3. Tobias C, Neubauer SC. Salt Marsh Biogeochemistry-An Overview. Coastal Wetlands. 2018; 539-596.

4. Howarth R. Microbial processes in salt-marsh sediments. In: Aquatic Microbiology: an Ecological Approach. 1993.

5. Thomas F, Giblin AE, Cardon ZG, Sievert SM. Rhizosphere heterogeneity shapes abundance and activity of sulfur-oxidizing bacteria in vegetated salt marsh sediments. Front Microbiol. 2014; 5: 309.

6. Hügler M, Huber H, Molyneaux SJ, Vetriani C, Sievert SM. Autotrophic CO2 fixation via the reductive tricarboxylic acid cycle in different lineages within the phylum Aquificae: Evidence for two ways of citrate cleavage. Environ Microbiol. 2007; 9(1): 81-92.

7. Sievert SM. Beyond the Calvin Cycle: Autotrophic Carbon Fixation in the Ocean. Ann. Rev. Mar. Sci. 2011; 3: 261-289

8. Middelburg JJ. Chemoautotrophy in the ocean. Geophys Res Lett. 2011; 38(24): 94-7.

9. Baltar F, Herndl GJ. Is dark carbon fixation relevant for oceanic primary production estimates? Biogeosciences. 2019; 16: 3793-3799.

10. Dyksma S, Bischof K, Fuchs BM, Hoffmann K, Meier D, Meyerdierks A. Ubiquitous Gammaproteobacteria dominate dark carbon fixation in coastal sediments. ISME J. 2016; 10(8): 1939-1953.

11. Peterson BJ, Howarth RW, Lipschultz F, Ashendorf D. An Alternative Interpretation of Stable Carbon Isotope Ratios and the Fate of Spartina alterniflora. OIKOS. 1979; 34: 173-7.

12. Vasquez-Cardenas D, Meysman FJR, Boschker HTS. A Cross-System Comparison of Dark Carbon Fixation in Coastal Sediments. Global Biogeochem Cycles. 2020; 34(2) .

13. Kappler U, Dahl C. Enzymology and molecular biology of prokaryotic sulfite oxidation. FEMS Microbiol Lett. 2001; 203(1): 1-9.

14. Pott AS, Dahl C. Sirohaem sulfite reductase and other proteins encoded by genes at the dsr locus of Chromatium vinosum are involved in the oxidation of intracellular sulfur. Microbiology. 1998; 144(7): 1881-94.

15. Friedrich CG, Rother D, Bardischewsky F, Quentmeier A, Fischer J. Oxidation of Reduced Inorganic Sulfur Compounds by Bacteria.pdf. Appl Environ Microbiol. 2001; 67(7): 2873-82.

16. Kelly DP, Shergill JK, Lu W-P, Wood AP. Oxidative metabolism of inorganic sulfur 
compounds by bacteria. 2012; 95-107.

17. Aoyagi T, Kimura M, Yamada N, Navarro RR, Itoh H, Ogata A. Dynamic transition of chemolithotrophic sulfur-oxidizing bacteria in response to amendment with nitrate in deposited marine sediments. Front Microbiol. 2015; 6: 1-12.

18. Bulseco AN, Vineis JH, Murphy AE, Spivak AC, Giblin AE, Tucker J. Metagenomics coupled with biogeochemical rates measurements provide evidence that nitrate addition stimulates respiration in salt marsh sediments. Limnol Oceanogr. 2020; 65: 321-39.

19. Bulseco AN, Giblin AE, Tucker J, Murphy AE, Sanderman J, Hiller-Bittrolff K. Nitrate addition stimulates microbial decomposition of organic matter in salt marsh sediments. Glob Chang Biol. 2019; 25(10): 3224-3241.

20. Eren AM, Esen ÖC, Quince C, Vineis JH, Morrison HG, Sogin ML. Anvi'o: an advanced analysis and visualization platform for 'omics data. PeerJ. https://dx.doi.org/10.7717/peerj.1319

21. Shaffer M, Borton MA, McGivern BB, Zayed AA, La Rosa SL, Solden LM. DRAM for distilling microbial metabolism to automate the curation of microbiome function. Nucleic Acids Res. 2020; 48(16): 8883-8900.

22. Fish JA, Chai B, Wang Q, Sun Y, Brown CT, Tiedje JM. FunGene: The functional gene pipeline and repository. Front Microbiol. 2013;4(OCT):1-14.

23. Cantarel BI, Coutinho PM, Rancurel C, Bernard T, Lombard V, Henrissat B. The Carbohydrate-Active EnZymes database (CAZy): An expert resource for glycogenomics. Nucleic Acids Res. 2009; 37: 233-238.

24. Overbeek R, Olson R, Pusch GD, Olsen GJ, Davis JJ, Disz T. The SEED and the Rapid Annotation of microbial genomes using Subsystems Technology (RAST). Nucleic Acids Res. 2014; Jan.

25. Nayfach S, Roux S, Seshadri R, Udwary D, Varghese N, Schulz F. A genomic catalog of Earth's microbiomes. Nat Biotechnol. 2021; 39(4): 499-509.

26. Chaumeil P-A, Mussig AJ, Hugenholtz P, Parks DH. GTDB-Tk: a toolkit to classify genomes with the Genome Taxonomy Database. Bioinformatics. 2019; 36(November 2019): 1925-7.

27. Parks DH, Chuvochina M, Waite DW, Rinke C, Skarshewski A, Chaumeil PA. A standardized bacterial taxonomy based on genome phylogeny substantially revises the tree of life. Nat Biotechnol. 2018; 36(10): 996.

28. Pritchard L, Glover RH, Humphris S, Elphinstone JG, Toth IK. Genomics and taxonomy in diagnostics for food security: Soft-rotting enterobacterial plant pathogens. Anal Methods. 2016; 8(1): 12-24.

29. Bushnell B. BBMap. sourceforge. 2021. Available from: https://sourceforge.net/projects/bbmap/

30. Li H, Handsaker B, Wysoker A, Fennell T, Ruan J, Homer N. The Sequence Alignment/Map format and SAMtools. Bioinformatics. 2009; 25(16): 2078-9.

31. Bowers RM, Kyrpides NC, Stepanauskas R, Harmon-Smith M, Doud D, Reddy TBK. Minimum information about a single amplified genome (MISAG) and a metagenome-assembled genome (MIMAG) of bacteria and archaea. Nat Biotechnol [Internet]. 2017; 35(8): 725-731.

32. Yuan H, Ge T, Chen C, O'Donnell AG, Wu J. Significant role for microbial autotrophy in the sequestration of soil carbon. Appl Environ Microbiol. 2012; 
644

78(7): 2328-36.

33. Hügler M, Gärtner A, Imhoff JF. Functional genes as markers for sulfur cycling and $\mathrm{CO} 2$ fixation in microbial communities of hydrothermal vents of the Logatchev field. FEMS Microbiol Ecol. 2010; 73(3): 526-37.

34. Zhou Z, Liu Y, Pan J, Cron BR, Toner BM, Anantharaman K. Gammaproteobacteria mediating utilization of methyl-, sulfur- and petroleum organic compounds in deep ocean hydrothermal plumes. ISME J. 2020;14(12):3136-48.

35. Bagnoud A, Chourey K, Hettich RL, De Bruijn I, Andersson AF, Leupin OX. Reconstructing a hydrogen-driven microbial metabolic network in Opalinus Clay rock. Nat Commun. 2016; 7: 1-10.

36. Kato S, Shibuya T, Takaki Y, Hirai M, Nunoura T, Suzuki K. Genome-enabled metabolic reconstruction of dominant chemosynthetic colonizers in deep-sea massive sulfide deposits. Environ Microbiol. 2018; 20(2): 862-77.

37. Koop-Jakobsen K, Gutbrod MS. Shallow Salt Marsh Tidal Ponds-An Environment With Extreme Oxygen Dynamics. Front Environ Sci. 2019; 7(October): 1-14.

38. Koop-Jakobsen K, Fischer J, Wenzhöfer F. Survey of sediment oxygenation in rhizospheres of the saltmarsh grass - Spartina anglica. Sci Total Environ. 2017; 589: 191-9.

39. Koop-Jakobsen K, Giblin AE. The effect of increased nitrate loading on nitrate reduction via denitirification and DNRA in salt marsh sediments. Limnology and Oceanography. 2010; 55(2): 789-802.

40. Lazar CS, Biddle JF, Meador TB, Blair N, Hinrichs KU, Teske AP. Environmental controls on intragroup diversity of the uncultured benthic archaea of the miscellaneous Crenarchaeotal group lineage naturally enriched in anoxic sediments of the White Oak River estuary (North Carolina, USA). Environ Microbiol. 2015; 17(7): 2228-38.

41. Valiela I, Teal JM. The nitrogen budget of a salt marsh ecosystem. Nature. 1979 Jan; 280(5724): 652-6.

42. Boschker HTS, De Brouwer JFC, Cappenberg TE. The contribution of macrophyte-derived organic matter to microbial biomass in salt-marsh sediments: Stable carbon isotope analysis of microbial biomarkers. Limnol Oceanogr. 1999; 44(2): 309-19.

43. Howes BL, Teal JM. Oxygen loss from Spartina alterniflora and its relationship to salt marsh oxygen balance. Oecologia. 1994; 97(4): 431-8.

44. Vasquez-Cardenas D, Quintana CO, Meysman FJR, Kristensen E, Boschker HTS. Species-specific effects of two bioturbating polychaetes on sediment chemoautotrophic bacteria. Mar Ecol Prog Ser. 2016; 549: 55-68.

45. Howarth RW, Teal JM. Sulfate reduction in a New England salt marsh. Limnol Oceanogr. 1979; 24(6): 999-1013.

46. Hines ME, Knollmeyer SL, Tugel JB. Sulfate reduction and other sedimentary biogeochemistry in a northern New England salt marsh. Limnol Oceanogr. 1989; 34(3): 578-590.

47. Kučera I, Sedláček V. Involvement of the cbb3-type terminal oxidase in growth competition of bacteria, biofilm formation, and in switching between denitrification and aerobic respiration. Microorganisms. 2020; 8(8): 1-11. 
690

691

692

693

694

695

696

697

698

699

700

701

702

703

704

705

706

707

708

709

710

711

712

713

714

715

716

717

718

719

720

721

722

723

724

725

726

727

728

729

730

731

732

733

734

735

48. Lennon JT, Aanderud ZT, Lehmkuhl BK, Schoolmaster DR. Mapping the niche space of soil microorganisms using taxonomy and traits. Ecology. 2012; 93(8): 1867-79.

49. Kearney M, Porter WP. Mapping the Fundamental Niche: Physiology, Climate , and the Distribution of a Nocturnal Lizard. Ecology. 2004; 85(11): 3119-3131.

50. King GM, Klug MJ, Wiegert RG CA. Relation of Soil Water Movement and Sulfide Concentration to Spartina alterniflora Production in a Georgia Salt Marsh.

Science. 1982; 218(1): 61-3.

51. Tyson GW, Chapman J, Hugenholtz P, Allen EE, Ram RJ, Richardson PM. Community structure and metabolism through reconstruction of microbial genomes from the environment. Nature. 2004; 428(6978): 37-43.

52. Bennett SA, Coleman M, Huber JA, Reddington E, Kinsey JC, Mclntyre C. Trophic regions of a hydrothermal plume dispersing away from an ultramafichosted vent-system: Von Damm vent-site, Mid-Cayman Rise. Geochemistry, Geophys Geosystems. 2013; 14(2): 317-27.

53. Coskun ÖK, Pichler M, Vargas S, Gilder S, Orsi WD. Linking Uncultivated Microbial Populations and Benthic Carbon Turnover by Using Quantitative Stable Isotope Probing. Appl Environ Microbiol. 2018; 84(18): e01083-18.

54. Lau MCY, Kieft TL, Kuloyo O, Linage-Alvarez B, Van Heerden E, Lindsay MR. An oligotrophic deep-subsurface community dependent on syntrophy is dominated by sulfur-driven autotrophic denitrifiers. Proc Natl Acad Sci. 2016; 113(49): 7927-36.

55. Quince C, Nurk S, Raguideau S, James R, Soyer OS, Kimberly Summers J. Metagenomics strain resolution on assembly graphs. Genome Biol. 2021; 22(214).

56. Han Y, Perner M. The globally widespread genus Sulfurimonas: Versatile energy metabolisms and adaptations to redox clines. Front Microbiol. 2015; 6(SEP): 117.

57. Tully BJ, Wheat CG, Glazer BT, Huber JA. A dynamic microbial community with high functional redundancy inhabits the cold, oxic subseafloor aquifer. ISME J. 2018; 12(1): 1-16.

58. Thomas F, Giblin AE, Cardon ZG, Sievert SM. Rhizosphere heterogeneity shapes abundance and activity of sulfur-oxidizing bacteria in vegetated salt marsh sediments. Front Microbiol. 2014; 5(JUN): 1-14.

59. Takai K, Campbell BJ, Cary SC, Suzuki M, Oida H, Nunoura T. Enzymatic and genetic characterization of carbon and energy metabolisms by deep-sea hydrothermal chemolithoautotrophic isolates of Epsilonproteobacteria. Appl Environ Microbiol. 2005; 71(11): 7310-7320.

60. Labrenz M, Grote J, Mammitzsch K, Boschker HTS, Laue M, Jost GI. Sulfurimonas gotlandica sp. nov., a chemoautotrophic and psychrotolerant epsilonproteobacterium isolated from a pelagic redoxcline, and an emended description of the genus Sulfurimonas. Int J Syst Evol Microbiol. 2013; 63(PART 11): 4141-8.

61. Ding J, Zhang $\mathrm{Y}$, Wang $\mathrm{H}$, Jian $\mathrm{H}$, Leng $\mathrm{H}$, Xiao X. Microbial community structure of deep-sea hydrothermal vents on the ultraslow spreading southwest Indian ridge. Front Microbiol. 2017;8(JUN):1-11.

62. Flood BE, Jones DS, Bailey J V. Sedimenticola thiotaurini sp. Nov., a sulfur- 
oxidizing bacterium isolated from salt marsh sediments, and emended descriptions of the genus sedimenticola and sedimenticola selenatireducens. Int $\mathrm{J}$ Syst Evol Microbiol. 2015; 65(8): 2522-30.

63. Marschall E, Jogler M, Henßge U, Overmann J. Large-scale distribution and activity patterns of an extremely low-light-adapted population of green sulfur bacteria in the Black Sea. Environ Microbiol. 2010; 12(5): 1348-62.

64. Repeta DJ, Simpson DJ, Jorgensen BB, Jannasch HW. Evidence for anoxygenic photosynthesis from the distribution of bacterio-chlorophylls in the Black Sea. Nature. 1989; 342(6245): 69-72.

65. Shen M, Li Q, Ren M, Lin Y, Wang J, Chen L. Trophic Status Is Associated With Community Structure and Metabolic Potential of Planktonic Microbiota in Plateau Lakes. Front Microbiol. 2019; 10(November): 1-15.

66. Thullner M, Regnier P, Van Cappellen P. Modeling microbially induced carbon degradation in redox-stratified subsurface environments: Concepts and open questions. Geomicrobiol J. 2007; 24(3-4): 139-55.

67. Jorgensen BB, Fossing $\mathrm{H}$, Wirsen $\mathrm{CO}$, Jannasch HW. Sulfide oxidation in the anoxic Black Sea chemocline. Deep Res Part A. 1991; 38(Suppl. 2A).

68. Knight VK, Nijenhuis I, Kerkhof LJ, Häggblom MM. Degradation of aromatic compounds coupled to selenate reduction. Geomicrobiol J. 2002; 19(1): 77-86.

Figure Legends:

Figure 1. Functional description of all MAGs recovered. From left to right 1) Functional clustering: the figure displays a hierarchical clustering of genomes based on a presence absence matrix of all functions identified by RAST. 2) The unique name for each MAG. The MAG naming convention includes the source of the MAG (N = enriched, $S=$ unenriched), the core ID $(1,2$, or 3$)$, the sediment depth $(S=$ shallow, $M=$ mid, $D=$ deep), the MAG (Bin) ID. A heat map displaying the detection of genes for each specific target function. The genes searched for each function are reported in Table S2. The functions in the heatmap are colored based on genes in each functional category: green $=$ Calvin-Benson-Bassham, pink = reductive TCA cycle, blue $=$ sulfur cycle, red = nitrogen cycle, dark blue = select carbohydrate utilization genes, and black = flagella . The variable heat displayed in the CBB and rTCA columns of the display represent the percentage of 9 and 10 genes used to evaluate the complete pathway respectively and can be found in Table S2. The functional categories for MAGs (Trophic group) classified as functionally capable of dark carbon fixation are displayed in the DCF group column and the heterotrophic canonical denitrifier groups are displayed in the Het group column. The functional groups in the legend for dark carbon fixation (DCF group) include Chlorobiaceae (CL), Magnetovibrio/Rhodospirallales (MR), Sedimenticola (S), Sulfurimonas (SUL), Thiohalomonadales/SZUA (TS) and undefined. The (Het group) includes two main clusters of MAGs, the Alteromonadacea/Nitrincolaceae/Kangiellacaea (ANK), and the koll-22 (k2). The GTDBTk taxonomy are presented in the last column.

Figure 2. A graphical summary of the functional potential of an A) Sedimenticola and B) Chlorobium MAG based on RAST and DRAM annotation. The Sedimenticola MAG 
782

represents a member of the TS group (Fig. 1) which uses the CBB carbon fixation pathway powered by denitrifying sulfur oxidation. Each reaction is indicated by an enzyme commission (EC) number or gene name within a green or yellow box. Green shading of the EC numbers indicates the presence of the gene in our MAG and a yellow background indicates the gene is absent. The substrates and products are indicated by a filled circle. Pathways include the potential primary carbon fixation pathway, metabolism of sulfur, nitrogen and key central carbon metabolisms of the cell.

Figure 3. Phylogenetic tree of Genomes from Earth's Microbiomes (GEM) and the flowthrough reactor (FTR) experiment MAGs. The tree at the center of the display was reconstructed using a minimum of 10 genes in a set of 26 ribosomal protein encoding genes. The first ring around the phylgenetic tree shows the phylum level assignment of each MAG according to the GTDB-Tk. The second ring indicates the source of the MAG (either FTR sediment from this experiment or from marine intertidal or estuary MAGs contained in the GEM database). The third ring shows the FTR C-fixation group assignment of the MAG as bars and the taxonomy of non-C-fixing (non chemoautotrophic) MAGs displayed as text. The circles displayed on the third ring represents the source of the FTR sample.

Figure 4. Relative abundance patterns of the 97 non-redundant set of MAGs in each sample. The relative abundance of each MAG is shown as a grey scale heatmap at the root of the display. Values represent log transformed average fold coverage that are also corrected for the number of reads in each sample to allow for comparison among MAGs and across samples. The heterotrophic and dark carbon fixation grouping of each MAG are annotated in the rings layers after the heat map, followed by the proportion of detection in each treatment, proportion of detection at each depth, MAG name, and finally layers indicated the depth where the MAG was recovered. We did not recover MAGs from the in-situ samples. Three of the MAGs show a number in parentheses following the name, this indicates the number of replicate genomes in the cluster. The full taxonomy for each MAG can be found in Table S3. The samples in the heat map are clustered based on Bray-Curtis distances of the corrected relative abundance. Metadata for each sample are provided as color bars beneath the hierarchical tree.

Figure 5. The proportion of gene clusters shared between one of the MAGs recovered in this study with reference genomes and other MAGs recovered within the same genus. The similarity in gene cluster number is based on the assignment of genes into gene clusters according to the Sedimenticola and Chlorobium pangenomic analysis. The color of the bar indicates the enviroment that the genome was recovered from and the taxonomy of the genome is indicated on the x-axis. An inverted triangle above the bar indicates that the representaitive genome was recovered from metagenomic data in this study. 
bioRxiv preprint doi: https://doi.org/10.1101/2021.08.24.457596; this version posted August 27, 2021. The copyright holder for this preprint (which was not certified by peer review) is the author/funder, who has granted bioRxiv a license to display the preprint in perpetuity. It is made Organic carbon utilization available under aCC-BY-NC-ND 4.0 International license.

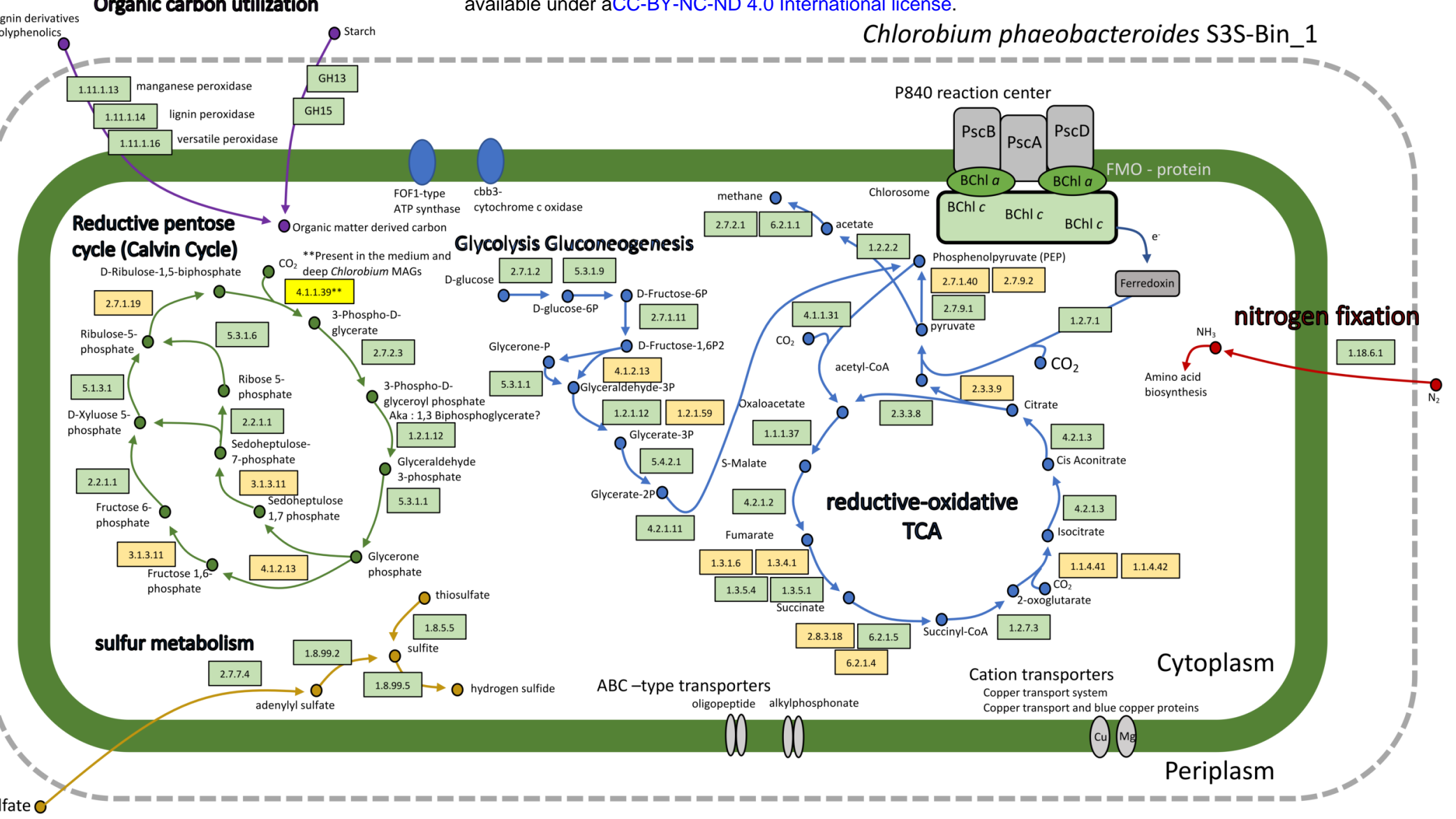
sulfate C

\section{Reductive pentose} cycle (Calvin Cycle)

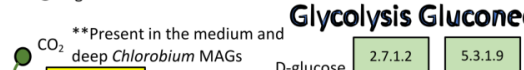

Fructose 6-

osphat

- $1,6 \mathrm{P} 2$ 


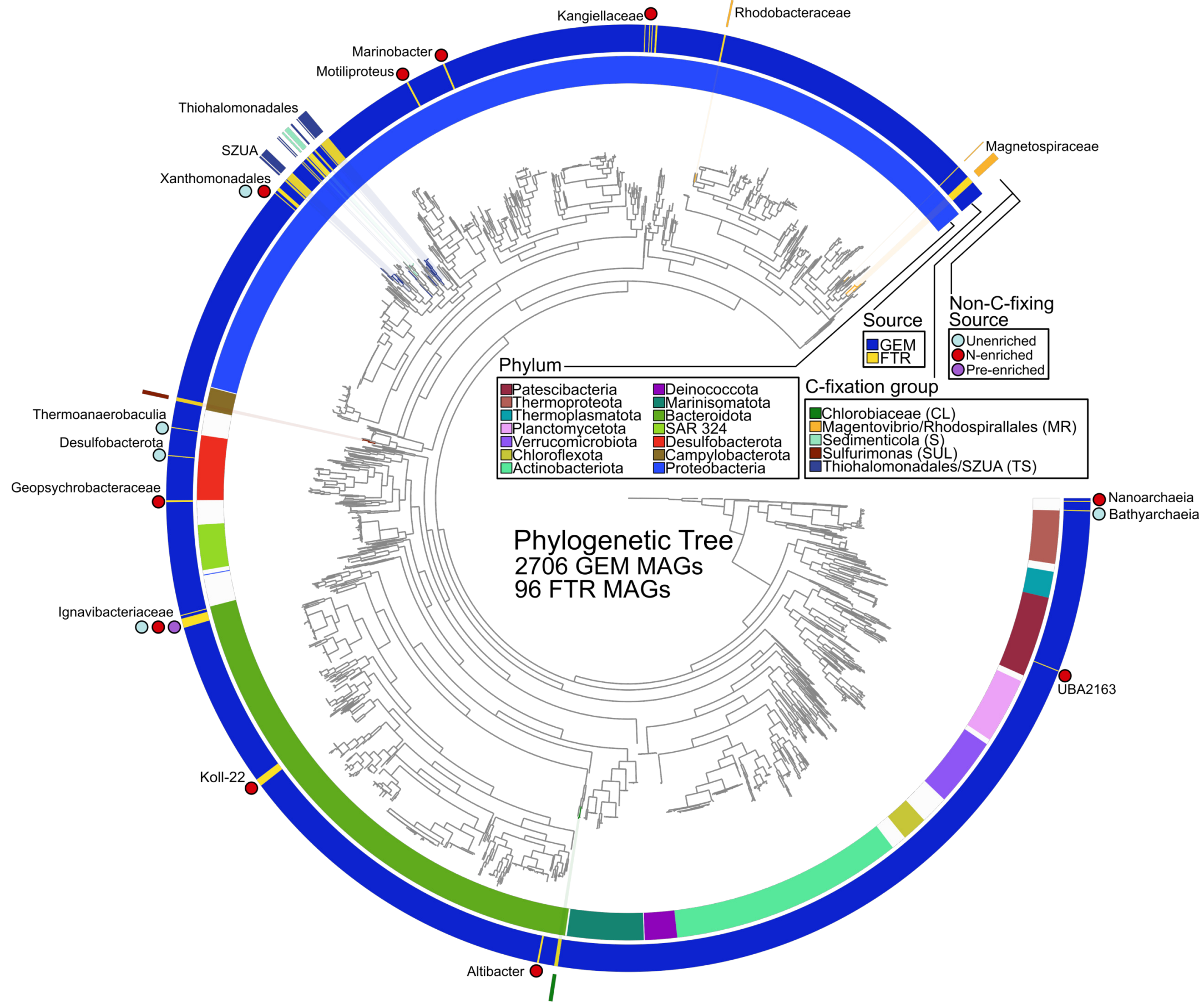

Figure 3. Phylogenetic tree of Genomes from Earth's Microbiomes (GEM) and the flowthrough reactor (FTR) experiment MAGs. The tree at the center of the display was reconstructed using a minimum of 10 genes in a set of 26 ribosomal protein encoding genes. The first ring around the phylgenetic tree shows the phylum level assignment of each MAG according to the GTDB-TK. The second ring indicates the source of the MAG (either FTR sediment from this experiment or from marine intertidal or estuary MAGs contained in the GEM database). The third ring shows the FTR C-fixation group assignment of the MAG as bars and the taxonomy of non-C-fixing (non chemoautotrophic) MAGs displayed as text. The circles displayed on the third ring represents the source of the FTR sample. 
bioRxiv preprint doi: https://doi.org/10.1101/2021.08.24.457596; this version posted August 27, 2021. The copyright holder for this preprint (which was not certified by peer review) is the author/funder, who has granted bioRxiv a license to display the preprint in perpetuity. It is made available under aCC-BY-NC-ND 4.0 International license.

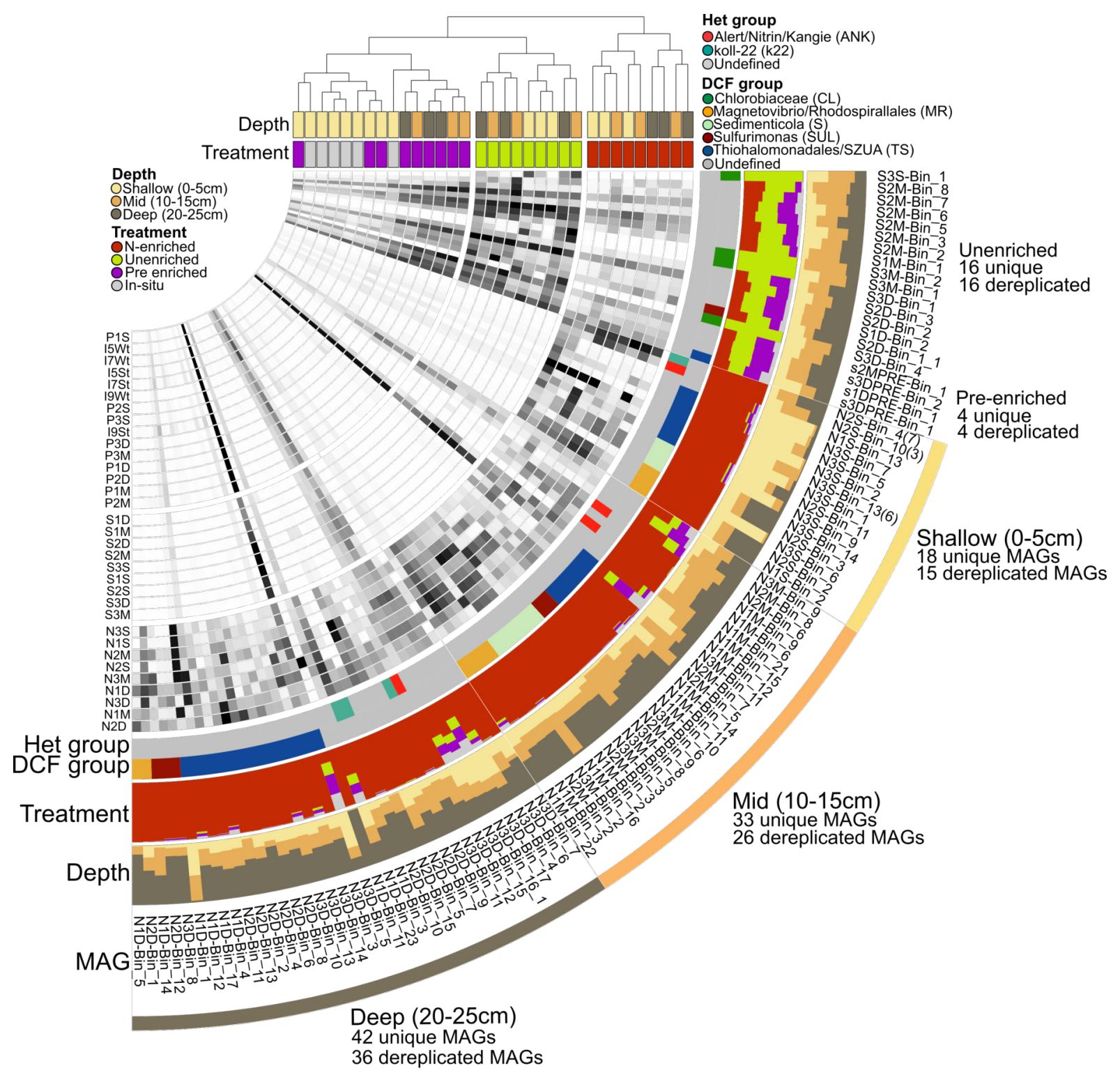

Figure 4. Relative abundance patterns of the 97 non-redundant set of MAGs in each sample. The relative abundance of each MAG is shown as a grey scale heatmap at the root of the display. Values represent log transformed average fold coverage that are also corrected for the number of reads in each sample to allow for comparison among MAGs and across samples. The heterotrophic and dark carbon fixation grouping of each MAG are annotated in the rings layers after the heat map, followed by the proportion of detection in each treatment, proportion of detection at each depth, MAG name, and finally layers indicated the depth where the MAG was recovered. We did not recover MAGs from the in-situ samples. Three of the MAGs show a number in parentheses following the name, this indicates the number of replicate genomes in the cluster. The full taxonomy for each MAG can be found in Table S3. The samples in the heat map are 


\section{Chlorobium}

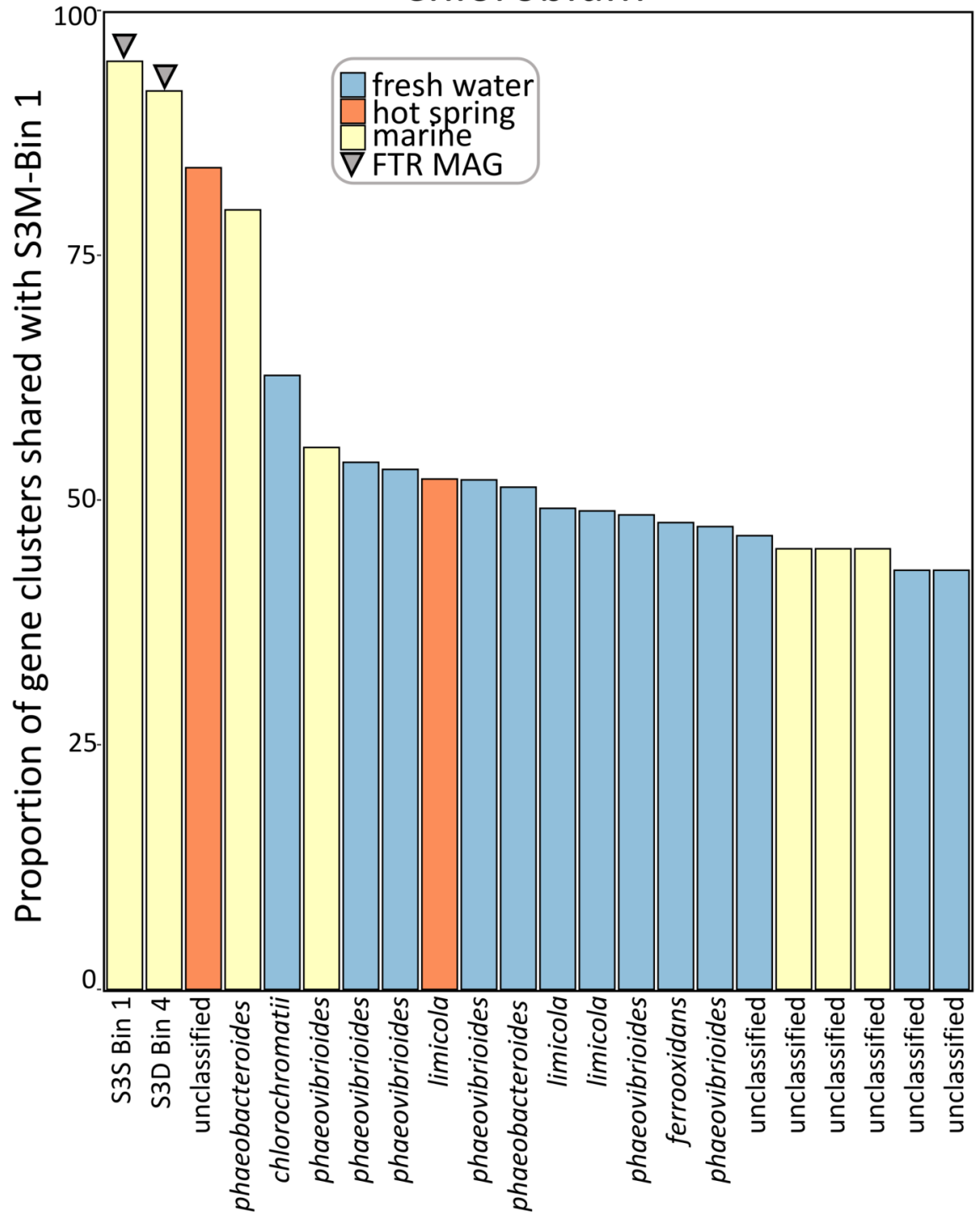

\section{Sedimenticola}

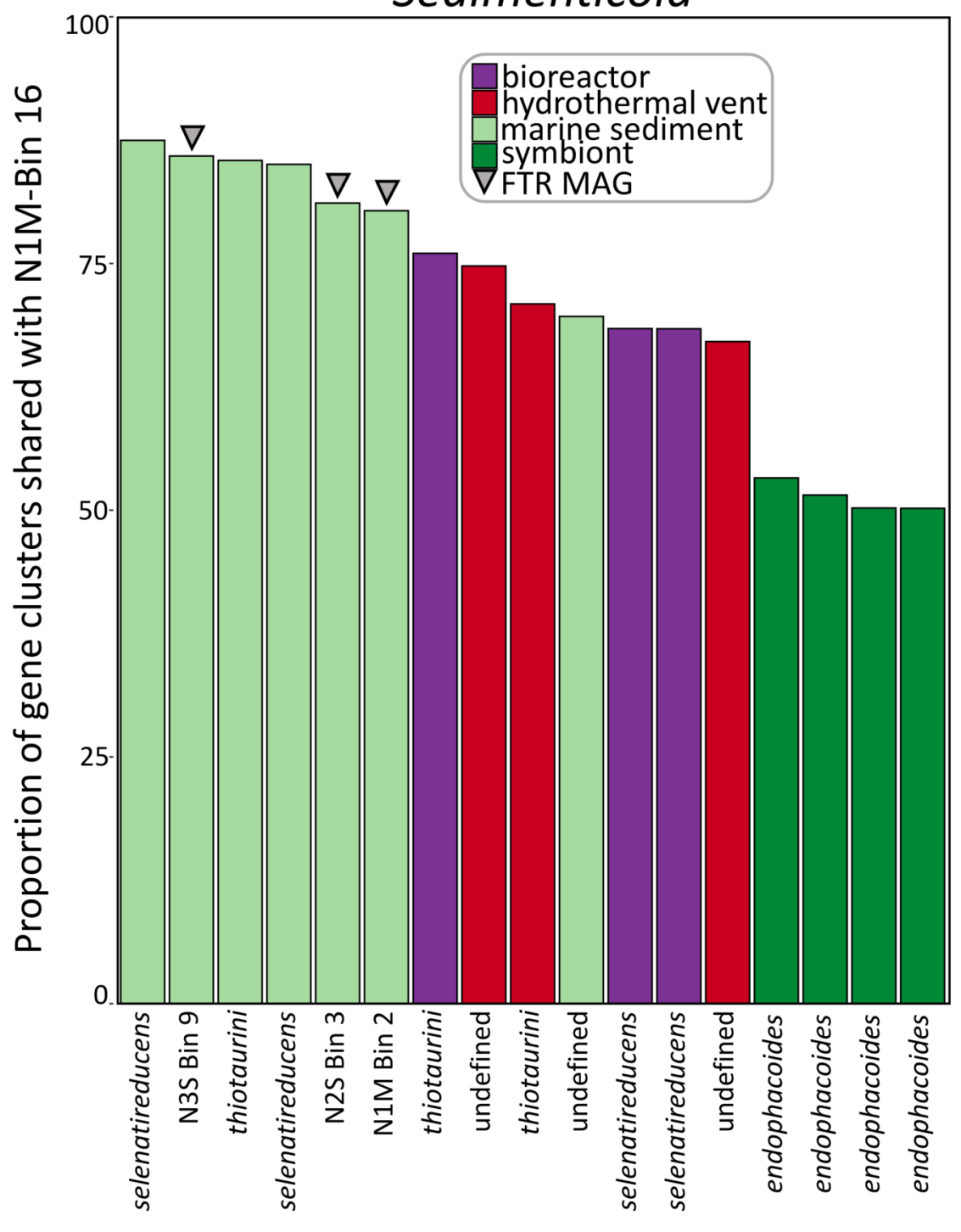

Figure 5. The proportion of gene clusters shared between one of the MAGs recovered in this study with reference genomes and other MAGs recovered within the same genus. The similarity in gene cluster number is based on the assignment of genes into gene clusters according to the Sedimenticola and Chlorobium pangenomic analysis. The color of the bar indicates the enviroment that the genome was recovered from and the taxonomy of the genome is indicated on the $\mathrm{x}$-axis. An inverted triangle above the bar indicates that the representaitive genome was recovered from metagenomic data in this study. 